\title{
Arabidopsis STO/BBX24 negatively regulates UV-B signaling by interacting with COP1 and repressing HY5 transcriptional activity
}

\author{
Lei Jiang ${ }^{1,3}$, Yan Wang ${ }^{2}$, Qian-Feng Li ${ }^{3}$, Lars Olof Björn ${ }^{1}$, Jun-Xian $\mathrm{He}^{3}$, Shao-Shan $\mathrm{Li}^{1}$
}

${ }^{I}$ Key Laboratory of Ecology and Environmental Science in Guangdong Higher Education, School of Life Science, South China Normal University, Guangzhou, Guangdong 510631, China; ${ }^{2}$ College of Life Science and Technology, Jinan University, Guangzhou, Guangdong 510632, China; ${ }^{3}$ State Key Laboratory of Agrobiotechnology and School of Life Sciences, The Chinese University of Hong Kong, Shatin, New Territories, Hong Kong SAR, China

UV-B (280-315 $\mathrm{nm})$ is an integral part of solar radiation and can act either as a stress inducer or as a developmental signal. In recent years, increasing attention has been paid to the low-fluence UV-B-induced photomorphogenic response and several key players in this response have been identified, which include UVR8 (a UV-B-specific photoreceptor), COP1 (a WD40-repeat-containing RING finger protein), HY5 (a basic zipper transcription factor), and RUP1/2 (two UVR8-interacting proteins). Here we report that Arabidopsis SALT TOLERANCE (STO/BBX24), a known regulator for light signaling in plants, defines a new signaling component in UV-B-mediated photomorphogenesis. The $b b x 24$ mutant is hypersensitive to UV-B radiation and becomes extremely dwarfed under UV-B treatment. By contrast, BBX24 overexpression transgenic lines respond much more weakly to UV-B than the $b b x 24$ and wild-type plants. BBX24 expression is UV-B-inducible and its accumulation under UV-B requires COP1. Co-immunoprecipitation experiments indicate that BBX24 interacts with COP1 in planta upon UV-B illumination. Moreover, BBX24 interacts with HY5 and acts antagonistically with HY5 in UV-B-induced inhibition of hypocotyl elongation. Furthermore, BBX24 attenuates UV-B-induced HY5 accumulation and suppresses its transcription-activation activity. Taken together, our results reveal a previously uncharacterized function of the light-regulated BBX24 in UV-B responses and demonstrate that BBX24 functions as a negative regulator of photomorphogenic UV-B responses by interacting with both COP1 and HY5. The UV-B-inducible expression pattern and its suppression of HY5 activity suggest that BBX24 could be a new component of the feedback regulatory module of $\mathrm{UV}$-B signaling in plants.

Keywords: UV-B; photomorphogenesis; STO/BBX24; COP1; HY5; Arabidopsis thaliana

Cell Research (2012) 22:1046-1057. doi:10.1038/cr.2012.34; published online 13 March 2012

\section{Introduction}

Ultraviolet-B (UV-B) radiation $(280-315 \mathrm{~nm})$ has great influence on biological processes, and does not merely act as a stress stimulus but can also, below the stress level, serve as an environmental signal to directly control

Correspondence: Jun-Xian $\mathrm{He}^{\mathrm{a}}$, Shao-Shan $\mathrm{Li}^{\mathrm{b}}$

${ }^{\mathrm{a}}$ Fax: +852-2603-6382

E-mail: jxhe@cuhk.edu.hk

${ }^{\mathrm{b}}$ Fax: +86-20-8521-1555

E-mail: lishsh@scnu.edu.cn

Received 25 August 2011; revised 27 November 2011; accepted 8 December 2011; published online 13 March 2012 growth and development [1]. Low levels of UV-B radiation cause certain photomorphogenic responses, including inhibition of hypocotyl elongation and root growth [2]. These UV-B photomorphogenic responses are mechanistically different from UV-B damage responses and are believed to be mediated by a UV-B-specific photoreceptor $[3,4]$. Recently, a UV-B photoreceptor has been identified in Arabidopsis, i.e., the $\beta$-propeller protein UV RESISTANCE LOCUS8 (UVR8) signaling through a pathway that involves the basic zipper (bZIP) transcription factor ELONGATED HYPOCOTYL5 (HY5) and the E3 ubiquitin ligase CONSTITUTIVELY PHOTOMORPHOGENIC 1 (COP1) [5].

UVR8 has sequence similarity to the human guanine 
nucleotide exchange factor REGULATOR OF CHROMATIN CONDENSATION1 (RCC1) [6]; however, it differs from RCC1 in activity and function [7]. UVR8 acts specifically to mediate plant responses to UV-B, such as suppression of hypocotyl elongation [8] and induction of UV-B responsive gene expression [9]. Recently, UVR8 was shown by biochemical studies to be a UV-B-specific photoreceptor [5]. In the absence of UV-B signal, UVR8 is present as homodimers that are capable of perceiving UV-B, probably by a tryptophan-based mechanism; absorption of UV-B induces instant monomerization and activation of the photoreceptor, followed by interaction with COP1 to relay the signal [5].

COP1 is a key negative regulator of light signaling [10]. It represses photomorphogenesis in darkness and acts as an E3 ubiquitin ligase, targeting different light-promoted transcription factors (such as HY5) for degradation in the dark [11]. The function of COP1 as an E3 ubiquitin ligase is impeded by light, resulting in activation of positive transcription factors to promote light response [10]. However, COP1 is a critical positive regulator of UV-B responses, contrasting to its function in light signaling [12]. HY5 is another important component shared by light and UV-B signaling. It is a bZIP transcription factor that positively regulates light-responsive gene expression [11], and it is required for plant survival under UV-B radiation [12]. COP1 is required for UV-B-induced $H Y 5$ gene activation, and both COP1 and HY5 proteins accumulate in the nucleus under UV-B [12], suggesting that HY5 is not the target of COP1 for degradation in UV-B signaling. It is proposed that COP1 may function to remove a negative regulator of UV-B responses [12], but no such component has been described.

SALT TOLERANCE (STO) is a B-box type zinc finger protein with sequence similarities to CONSTANS [13]. It is also called BBX24 according to the nomenclature for the B-box transcription factors [14], and we use this nomenclature in the rest of the paper. Despite that BBX24 was originally identified from Arabidopsis as a protein conferring salt tolerance in yeast [15], the majority of its characterized functions are involved in light signaling in Arabidopsis [16]. BBX24 acts as a negative regulator in phytochrome and blue-light signaling. The bbx24 mutant exhibits shorter hypocotyl and higher $C H S$ gene expression under all light conditions [16]. The BBX24 protein is transiently induced by light and its proper functioning in light responses depends on its interaction with COP1 [17].

Our previous research has revealed that RADICALINDUCED CELL DEATHI (RCDI) is involved in UV-B signaling. The expression of COP1-regulated UV-B responsive genes was enhanced in $r c d 1-1$ mutant under
UV-B radiation, indicating that $R C D 1$ negatively regulates UV-B response [18]. Interestingly, RCD1 interacts with $\mathrm{STO} / \mathrm{BBX} 24$ in vitro [19]. These findings prompted us to test whether STO/BBX24 functions in UV-B photomorphogenic responses. In the course of this study, we discovered that BBX24 negatively regulates UV-B signaling and directly interacts with both COP1 and HY5. Thus, our study identified BBX24 as a new signaling component in UV-B signaling and indicated that the functional mechanism of BBX24 in UV-B response is distinct from that in light signaling.

\section{Results}

BBX24 negatively regulates $U V-B$ responses in both Arabidopsis shoots and roots

Two widely characterized phenotypes in UV-B photomorphogenic responses are inhibition of hypocotyl growth and accumulation of anthocyanins [4]. To investigate whether BBX24 is involved in UV-B signaling, we examined these phenotypes in the $b b \times 24$ mutant and BBX24 overexpression transgenic lines (35S::BBX24GFP, Supplementary information, Figure S1) under UV-B radiation. When the seedlings were grown for 5 days under the weak white light $\left(10 \mu \mathrm{mol} \mathrm{m} \mathrm{m}^{-2} \mathrm{~s}^{-1}\right.$ PAR $)$ supplemented with narrow-band UV-B, they all responded to UV-B radiation with shorter hypocotyls and higher contents of anthocyanins compared to the mocktreated seedlings (Figure 1A-1C). However, the responses of the $b b \times 24$ mutant are much greater than the three 35S::BBX24-GFP overexpression lines and their wildtype control (Col) (Figure 1A-1C). These results suggest that BBX24 functions negatively in UV-B photomorphogenic responses.

To test whether the UV-B-induced short hypocotyl phenotype of the bbx24 mutant is mediated by any of the known photoreceptors (cryptochromes, phototropins, or phytochromes), we crossed $b b \times 24$ to a number of photoreceptor mutants, including phyA phyB, phot 1 phot 2 , and cryl cry2. Analysis of crosses homozygous for $b b \times 24$ and the various photoreceptor genes showed that none of these mutations affected the $b b \times 24$ shorter hypocotyl phenotype under UV-B (Supplementary information, Figure S2), suggesting that UV-B-induced inhibition of hypocotyl elongation is not mediated by any of these photoreceptors.

In addition to inhibiting hypocotyl elongation, we found that UV-B also affects root development. To determine whether BBX24 is involved in UV-B-regulated plant root growth, the primary root length of 7-day-old seedlings of the wild type and bbx24 mutant was determined under various light conditions, including white 
A

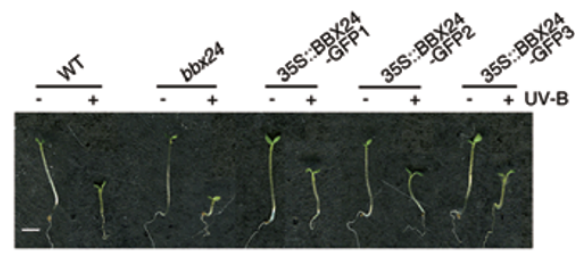

D
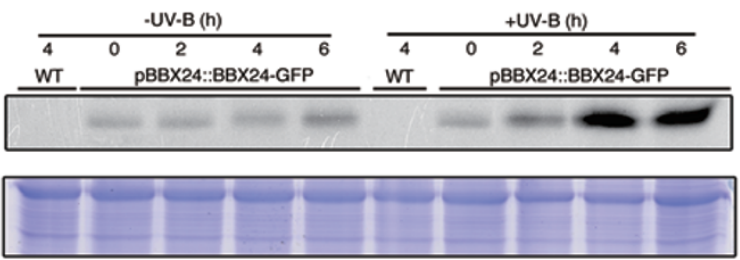

B

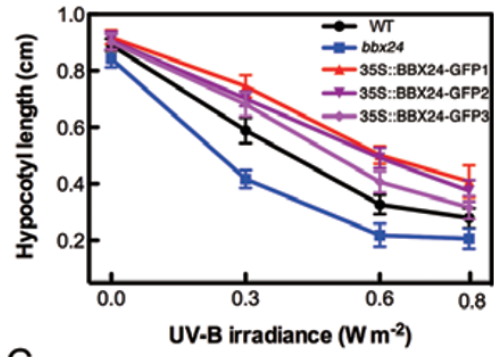

C

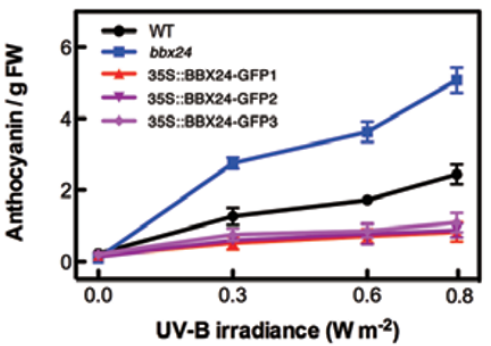

E

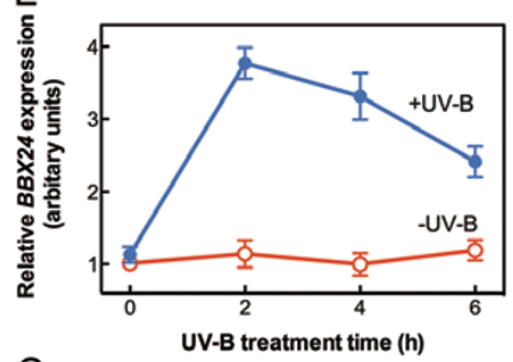

G

UV-B treatment time (h)

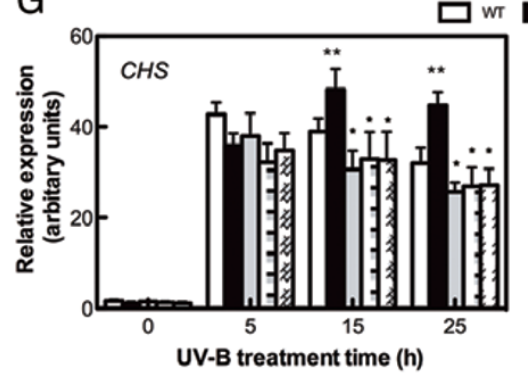

F

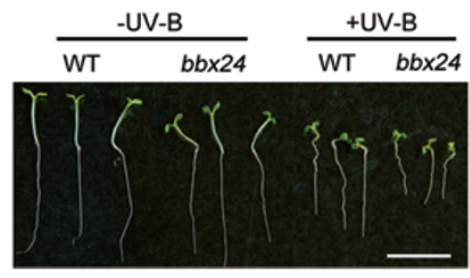

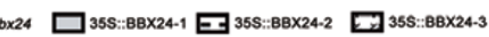

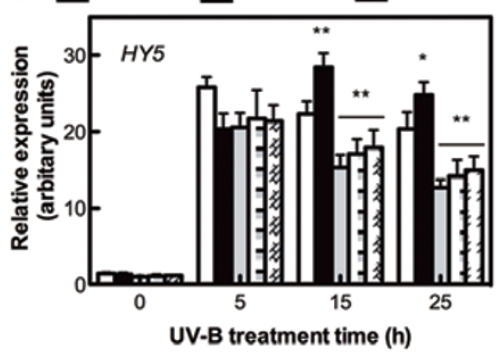

Figure $1 \mathrm{BBX} 24$ is a negative regulator of UV-B signaling in Arabidopsis. (A) Hypocotyl phenotype of 5-day-old wild type (WT), $b b \times 24$ mutant and BBX24-overexpression (35S::BBX24-GFP) transgenic lines under continuous UV-B radiation $\left(0.6 \mathrm{~W} \mathrm{~m}^{-2}\right)$. Scale bar: $0.2 \mathrm{~cm}$. (B) Quantification of hypocotyl length of seedlings in A under various UV-B radiations. (C) Anthocyanin content in $b b \times 24$ and the 35S::BBX24-GFP transgenic plants. Data shown in B and $\mathbf{C}$ are means \pm SEM of three independent replications. The hypocotyl length and anthocyanin content of WT are significantly different with mutant and overexpression lines after UV-B treatment (Student's $t$-test). (D) Induction of BBX24 protein by UV-B. Wild type and pBBX24::BBX24-GFP transgenic plants were grown under white light for 5 days, then transferred to supplementary UV-B radiation for various times indicated. (E) Effects of UV-B on BBX24 gene expression detected by real-time RT-PCR. Data shown are mean \pm SEM of four independent replications. (F) Shorter primary root of bbx24 under UV-B radiation. Wild type and bbx24 were grown under various light conditions for 7 days (light/dark, 16/8 h). (G) Relative expression of CHS and HY5 genes in wild type, bbx24 and 35S::BBX24-GFP detected by real-time PCR. The plants were grown under the same condition as in $\mathbf{D}$ and the relative expression of transcripts was quantified by real-time RT-PCR. All the data shown are means \pm SEM of three independent replications. Statistically significant differences of $b b \times 24$ or 35 S::BBX24-GFP relative to wild type are indicated by a double asterisk $(P$ $<0.01)$ or a single asterisk $(P<0.05)$.

light $\left(80 \mu \mathrm{mol} \mathrm{m} \mathrm{m}^{-2} \mathrm{~s}^{-1}\right)$, blue light $\left(9 \mu \mathrm{mol} \mathrm{m}{ }^{-2} \mathrm{~s}^{-1}\right)$, red light $\left(45 \mu \mathrm{mol} \mathrm{m} \mathrm{s}^{-1}\right)$, far-red light $\left(20 \mu \mathrm{mol} \mathrm{m}{ }^{-2} \mathrm{~s}^{-1}\right)$, and UV-B $\left(0.6 \mathrm{~W} \mathrm{~m}^{-2}\right)$. Compared to other light conditions, UV-B treatment caused significant arrest of primary root elongation in Arabidopsis and the arrest was enhanced in the $b b \times 24$ mutant at as early as 5 days of the UV-B treatment (Figure $1 \mathrm{~F}$ and Supplementary information, Figure S3). No difference in primary root length was found between the wild type and $b b \times 24$ under other light conditions, indicating that BBX24 is also a negative regulator of UV-B inhibition of primary root growth.
Both BBX24 mRNA and protein are UV-B-inducible and misexpression of $B B X 24$ affects $U V$-B-responsive gene expression

To determine whether $B B X 24$ is regulated by UV-B, we examined both its mRNA and protein levels under UV-B treatment. It is apparent from quantitative RT-PCR results that the BBX24 mRNA was increased significantly after $2 \mathrm{~h}$ of UV-B treatment, followed by a gradual decrease after $6 \mathrm{~h}$ (Figure 1E). For protein determination, we generated transgenic plants expressing the BBX24GFP fusion protein under the control of a $35 \mathrm{~S}$ or the $B B X 24$ promoter and the BBX24-GFP protein expres- 
sion level was detected with an anti-GFP antibody (Santa Cruz Biotechnology, CA, USA). While the 35S::BBX24GFP transgenic plants have weaker UV-B responses than their wild-type control (Figure 1A-1C), the phenotype of the BBX24::BBX24-GFP plants is not distinguishable from wild type under the UV-B treatment (Supplementary information, Figure S4), suggesting that the BBX24-GFP protein functions just like the wild-type BBX24. Western blot analyses using both the 35S::BBX24-GFP and BBX24::BBX24-GFP plants showed that the BBX24GFP protein level did not increase until $4 \mathrm{~h}$ of the UV-B treatment (Figure 1D, Supplementary information, Figure S6), and decreased again when UV-B treatment was terminated (Supplementary information, Figures S5 and S6). These results indicate that $S T O / B B X 24$ gene expression is affected by UV-B at both transcriptional and posttranscriptional levels.

Further, we performed quantitative expression analyses on two reported UV-B responsive genes CHS and HY5 under UV-B. Wild type, bbx24 and 35S::BBX24 transgenic plants were grown first under a weak white light $\left(10 \mu \mathrm{mol} \mathrm{m} \mathrm{m}^{-2} \mathrm{~s}^{-1}\right)$ for 5 days and then transferred to supplementary UV-B for the times indicated. After $5 \mathrm{~h}$ of UV-B treatment, the expression of CHS and HY5 showed no significant difference between plants of different genotypes. However, after prolonged UV-B treatment (15 to $25 \mathrm{~h}$ ), the bbx24 mutant showed increased CHS and HY5 induction, whereas 35S::BBX24-GFP lines exhibited reduced induction (Figure $1 \mathrm{G}$ ). In addition, four other UV-B responsive genes - ELIP1, PHR1, SIG5, and $W A K L 8$ were shown to have increased and decreased expression in $b b \times 24$ mutant and 35S::BBX24, respectively, after UV-B treatment (Supplementary information, Figure S7). This pattern of UV-B-responsive gene expression further supports the notion that $B B X 24$ functions as a negative regulator in UV-B signaling.

BBX24 interacts with COP1 in plants and acts downstream of $C O P 1$ in $U V-B$ signaling

Previous studies have shown that BBX24 interacts with COP1 in yeast [20], and COP1 acts as a negative component in light signaling [10] but a positive regulator in UV-B response [12]. Thus, it is of great interest to evaluate a direct physical interaction between BBX24 and COP1 in plants. To do this, we generated transgenic plants expressing the full-length BBX24 and GFP fusion protein under the control of a $35 \mathrm{~S}$ promoter, and the in vivo interaction between BBX24 and COP1 was determined by the co-immunoprecipitation (Co-IP) method. The plants were crossed with the cop1-4 mutant to obtain the BBX24-GFP overexpression lines in the cop1 background, which together with the $35 \mathrm{~S}:$ GFP plants, were used as negative controls. All testing materials were grown under weak white light for 5 days and then transferred to supplementary UV-B for $24 \mathrm{~h}$. BBX24 and its interacting partners were immunoprecipitated from these seedlings by a GFP antibody and BBX24-COP1 interaction was detected by using a COP1 antibody. A band with expected mobility of COP1 was clearly detected from the immunoprecipitates of the 35S::BBX24-GFP plants under UV-B treatment but not from that of the non-UV-B treated plants (Figure 2A). Consistently, no COP1 band was detected from immunoprecipitates of UV-B-treated cop 1-4 35S::BBX24-GFP and 35S::GFP plants (Figure $2 \mathrm{~A})$. These Co-IP results strongly suggest that BBX24 directly interacts with COP1 in planta.

To test whether COP1 is required for the degradation of BBX24, we compared the BBX24-GFP protein level in 5-day-old pBBX24::BBX24-GFP transgenic plants (either in the WT or copl-4 background) grown under darkness or UV-B, before and after treatment with MG132, a proteasome inhibitor. The results (Figure 2B) revealed a dramatic increase of BBX24-GFP accumulation in the dark-grown seedlings treated with MG132, indicating that the BBX24 protein is regulated by the $26 \mathrm{~S}$ proteasome-mediated protein degradation pathway in the dark. However, loss-of-function of COP1 (cop1-4) resulted in accumulation of BBX24 even without MG132 treatment in the dark (Figure 2B), suggesting that COP1 might be responsible for the degradation of BBX24 in darkness. Surprisingly, when the dark-grown seedlings were transferred to UV-B, the BBX24 protein accumulation was significantly increased, and MG132 treatment had little effect on UV-B-induced BBX24 accumulation (Figure 2B). Furthermore, the UV-B-induced BBX24 accumulation was reduced in the cop 1-4 background (Figure 2B and Supplementary information, Figure S8). These results indicate that the $26 \mathrm{~S}$ proteasome does not mediate BBX24 degradation under UV-B and that COP1 appears to be required for UV-B-induced BBX24 accumulation.

To further investigate how COP1 and BBX24 function together to mediate UV-B response, we crossed the cop14 mutant to the bbx24 mutant and the 35S::BBX24-GFP overexpression lines to examine the genetic relationship between COP1 and BBX24. The relative hypocotyl length $(\%$ of $+\mathrm{UV}-\mathrm{B} /-\mathrm{UV}-\mathrm{B})$ and relative anthocyanin accumulation (+UV-B/-UV-B) were used to evaluate the responses to UV-B. The weak UV-B response of the cop 1-4 hypocotyl was significantly suppressed by the bbx24 mutation, which resulted in similar hypocotyl length of the cop1-4 bbx24 double mutants to $b b \times 24$ under UV-B (Figure 2C). Meanwhile, the defect of UV-Binduced anthocyanin accumulation in cop 1-4 was also significantly suppressed by $b b x 24$ (Figure 2D), again 
indicating that $\mathrm{BBX} 24$ is a negative regulator of plant $\mathrm{UV}-\mathrm{B}$ responses and that $\mathrm{BBX} 24$ acts downstream of COP1 in UV-B signaling.

BBX24 interacts and functions antagonistically with HY5 in UV-B signaling

It has been shown previously that two BBX24 homologs, STH2/BBX21 and STH3/BBX22, interact with HY5 in yeast $[21,22]$. The essential role of HY5 in UV-B signaling [23] encouraged us to examine whether BBX24 physically interacts with HY5. Full-length HY5 fused with the GAL4-DNA-binding domain interacted strongly with the BBX24 fused with the GAL4-activation domain in the yeast two-hybrid system (Figure 3A). However, deletion of the bZIP domain in HY5 resulted in dramatic reduction of BBX24-HY5 interaction, indicating that the bZIP domain is required for HY5's interaction with BBX24 (Figure 3A). This interaction was further confirmed by co-immonuprecipitation assay using transgenic plants co-expressing the HY5 and BBX24-GFP proteins. Protein extracts from testing plants (see Figure 3B) were immunoprecipitated by a GFP antibody and the precipitated proteins were analyzed by western blotting using a HY5 antibody. Bands of HY5 were observed from the immunoprecipitates of 35S::BBX24-GFP plants grown with or without UV-B, but not from the hy5-215 35S::BBX24-GFP plants (Figure 3B), indicating BBX24 interacts with HY5 in vivo.

We further crossed the hy5-215 mutant with $b b \times 24$ and 35S::BBX24-GFP plants to investigate the genetic relationship between HY5 and BBX24. These plants were grown for 5 days under the weak white light (10
A

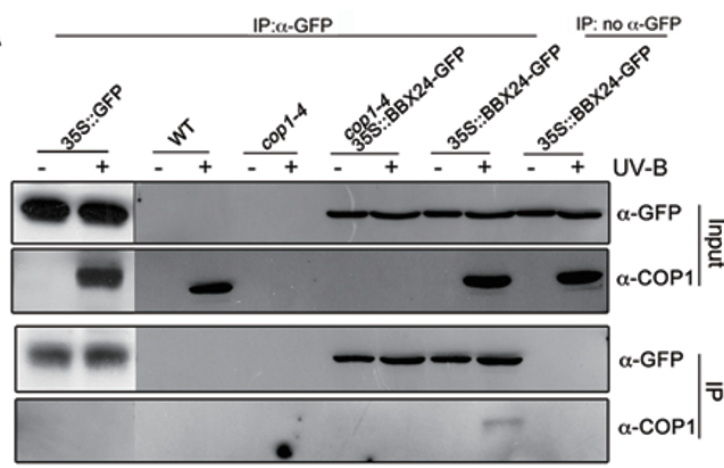

C

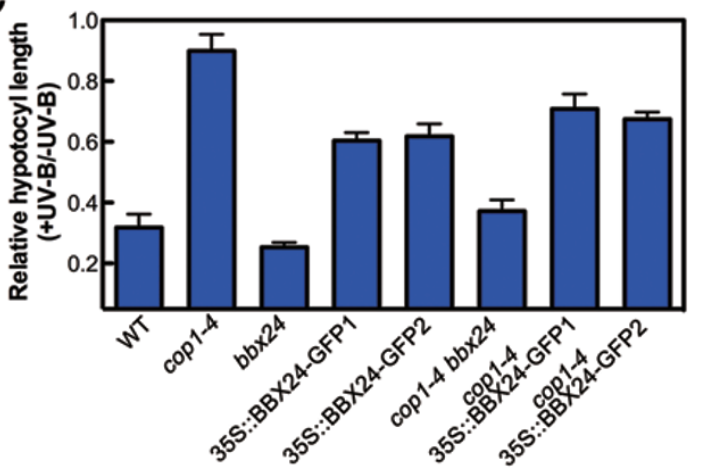

B
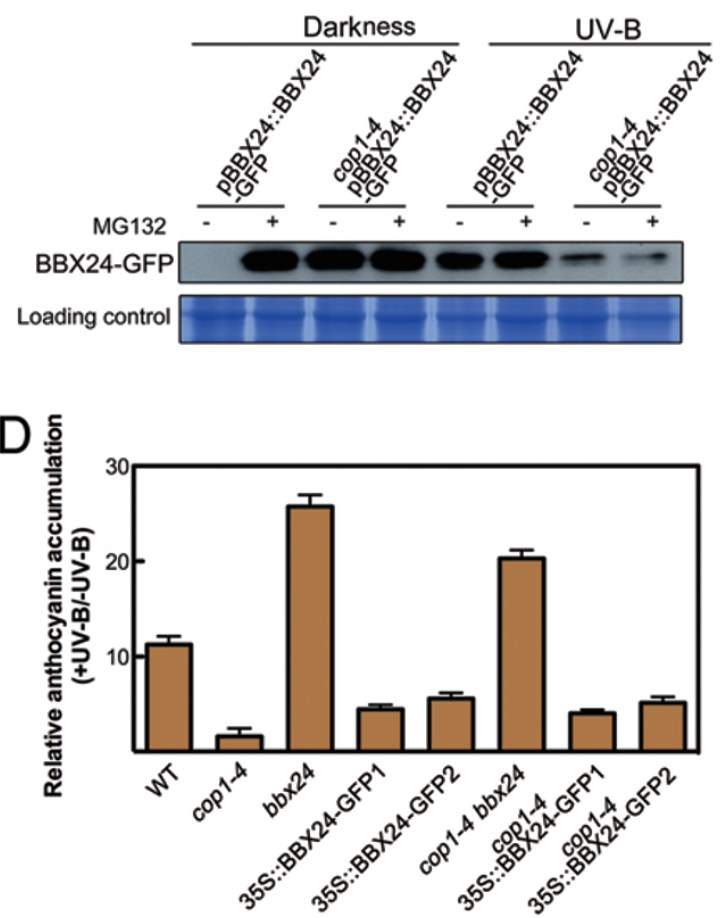

Figure 2 Interaction of BBX24 and COP1 in plants. (A) Co-IP detection of in vivo interaction between COP1 and BBX24-GFP. Five-day-old seedlings were UV-B irradiated (+UV-B) or mock treated (-UV-B) for $24 \mathrm{~h}$. Protein extracts from the wild type and various transgenic seedlings were immunoprecipitated with a GFP antibody, separated on $10 \%$ SDS-PAGE, blotted onto PVDF membranes, and detected with either a GFP or COP1 antibodies. 'Input' refers to the starting protein extracts used for IP reactions. The protein sample from 35S::GFP was used as a negative control of the Co-IP experiments. (B) COP1 mediates BBX24 degradation in the dark but is required for the BBX24 stability under UV-B. Five-day-old dark-grown seedlings were treated with MG132 (+) or DMSO (-) for 6 h under either darkness or UV-B. Total protein was extracted and subjected to immunoblot analysis with a GFP antibody. (C) Relative hypocotyl length of bbx24 and 35S::BBX24-GFP plants in the wild type and cop1-4 background under UV-B treatment. (D) Relative anthocyanin accumulation of bbx24 and 35S::BBX24-GFP plants in the wild type and cop1-4 background during UV-B treatment. Data shown are means \pm SEM of three independent replications. 

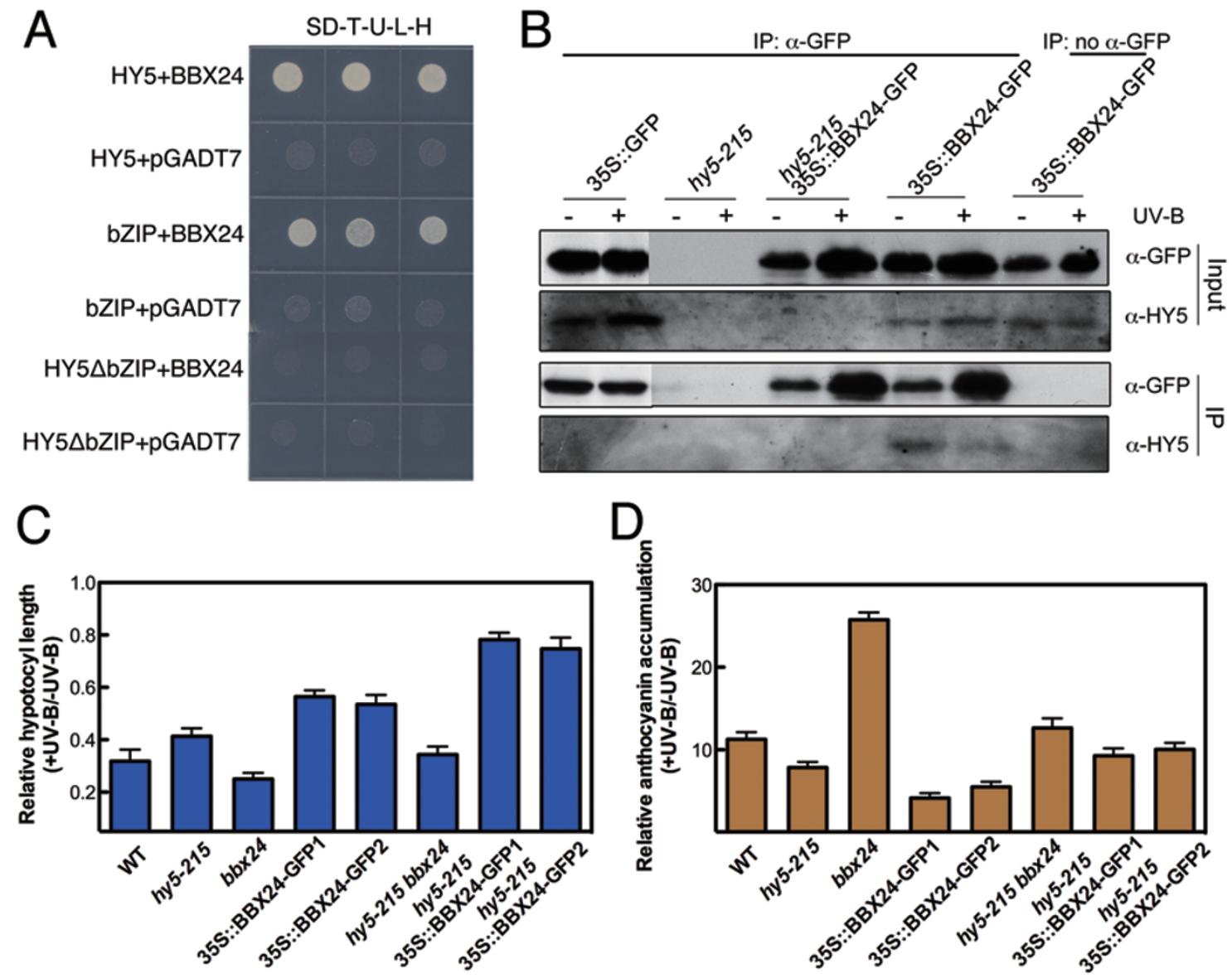

Figure 3 Interaction between BBX24 and HY5. (A) BBX24 interacts with HY5 in yeast. The yeast strain AH109 was cotransformed with a bait ( $p G B K T 7-H Y 5, p G B K T 7-H Y 5 \Delta b Z I P$ or $p G B K T 7-b Z I P)$ and a prey ( $p G A D T 7-B B X 24)$ construct. The empty prey vector was used as a negative control. Interactions of each test protein pairs were determined by growth on His selection medium. (B) Interaction of HY5 with BBX24 in vivo. Five-day-old seedlings were UV-B irradiated (+UV-B) or mock treated (-UV-B) for $24 \mathrm{~h}$ and Co-IP assays were performed as described in Figure 2. (C) Relative hypocotyl length of bbx24 and 35S::BBX24-GFP plants in the wild type and hy5-215 background under UV-B treatment. (D) BBX24 and HY5 antagonistically regulate anthocyanin accumulation in plants during UV-B treatment. All the data shown are means \pm SEM of three independent replications.

$\left.\mu \mathrm{mol} \mathrm{m} \mathrm{m}^{-2}\right)$ supplemented with UV-B $\left(0.6 \mathrm{~W} \mathrm{~m}^{-2}\right)$. Compared to the wild type, the hy5-215 mutant had a longer hypocotyl while $b b \times 24$ showed a shorter hypocotyl under UV-B. However, the hypocotyl length of the hy5-215 was partially suppressed by $b b \times 24$ in the hy5$215 b b \times 24$ double mutant under UV-B (Figure 3C). The same response pattern of the hy5-215 bbx24 double mutant was observed for anythocyanin accumulation (Figure 3D). All these genetic results indicate that BBX24 acts antagonistically with HY5 in UV-B signaling. However, when BBX24 was overexpressed in the hy5-215 mutant background, it enhanced the long hypocotyl phenotype of the mutant (Figure 3C), suggesting that BBX24 only partially functions through the HY5 signaling pathway or it has other target(s) in plants.
BBX24 suppresses the UV-B-induced HY5 accumulation and negatively modulates HY5's transcriptional activity

To further understand how BBX24 regulates UV-B signaling, we first determined whether BBX24 affects the UV-B-induced HY5 accumulation. Wild type, $b b \times 24$ and 35S::BBX24-GFP were grown under weak white light $\left(10 \mu \mathrm{mol} \mathrm{m} \mathrm{m}^{-2} \mathrm{~s}^{-1}\right)$ for 5 days, and then transferred to supplementary UV-B radiation $\left(0.6 \mathrm{~W} \mathrm{~m}^{-2}\right)$ for $5 \mathrm{~h}$. The UV-B-induced HY5 accumulation was significantly decreased in 35S::BBX24-GFP transgenic plants, while no difference between $b b \times 24$ and wild type was observed from this experiment (Figure 4A). This result indicates that BBX24 suppresses the UV-B-induced HY5 accumulation. Consistent with this result, we observed a reduced interaction of HY5 with BBX24-GFP protein in the Co- 
IP experiments under UV-B compared to UV-B condition (Figure 3B), which could be due to a reduced HY5 protein level caused by BBX24 overexpression.

Because HY5 is an essential transcription factor in UV-B responses [23], we then analyzed whether BBX24 can affect HY5's transcriptional activity in a transient assay under UV-B. For this purpose, HY5 was expressed as a fusion protein with the GAL4-DNA-binding domain under the control of a $35 \mathrm{~S}$ promoter (Figure 4B). The reporter construct consists of a minimal $35 \mathrm{~S}$ promoter with the GAL4-DNA binding site and a luciferase reporter gene. A Renilla luciferase gene under the control of a $35 \mathrm{~S}$ promoter was used as an internal control. These constructs were expressed transiently in Arabidopsis protoplasts, and luciferase activity was assayed after $6 \mathrm{~h}$ of supplementary UV-B treatment $\left(0.6 \mathrm{~W} \mathrm{~m}^{-2}\right)$. HY5 stimulated higher luciferase expression than that of the DNAbinding domain (DBD) control in UV-B-incubated proto- plasts, indicating that HY5 has the capability to function as a transcriptional activator in vivo (Figure 4C). When these constructs were transformed into the $b b \times 24$ protoplasts, luciferase expression induced by UV-B treatment was significantly increased, while it was decreased in the 35S::BBX24 protoplasts (Figure 4D). The yeast GAL4 DNA-binding domain (DBD) and transcription-activation domain (AD) fusion (DBDAD in pMN7), showed no such responsiveness to the UV-B (Figure 4D). These results indicate that BBX24 is capable of negatively regulating the transcriptional activity of HY5 in response to UV-B. Therefore, we hypothesize that BBX24 functions as a new repressor of UV-B signaling by directly impinging on the HY5 protein activity.

\section{Discussion}

UV-B is part of the solar radiation and functions as an
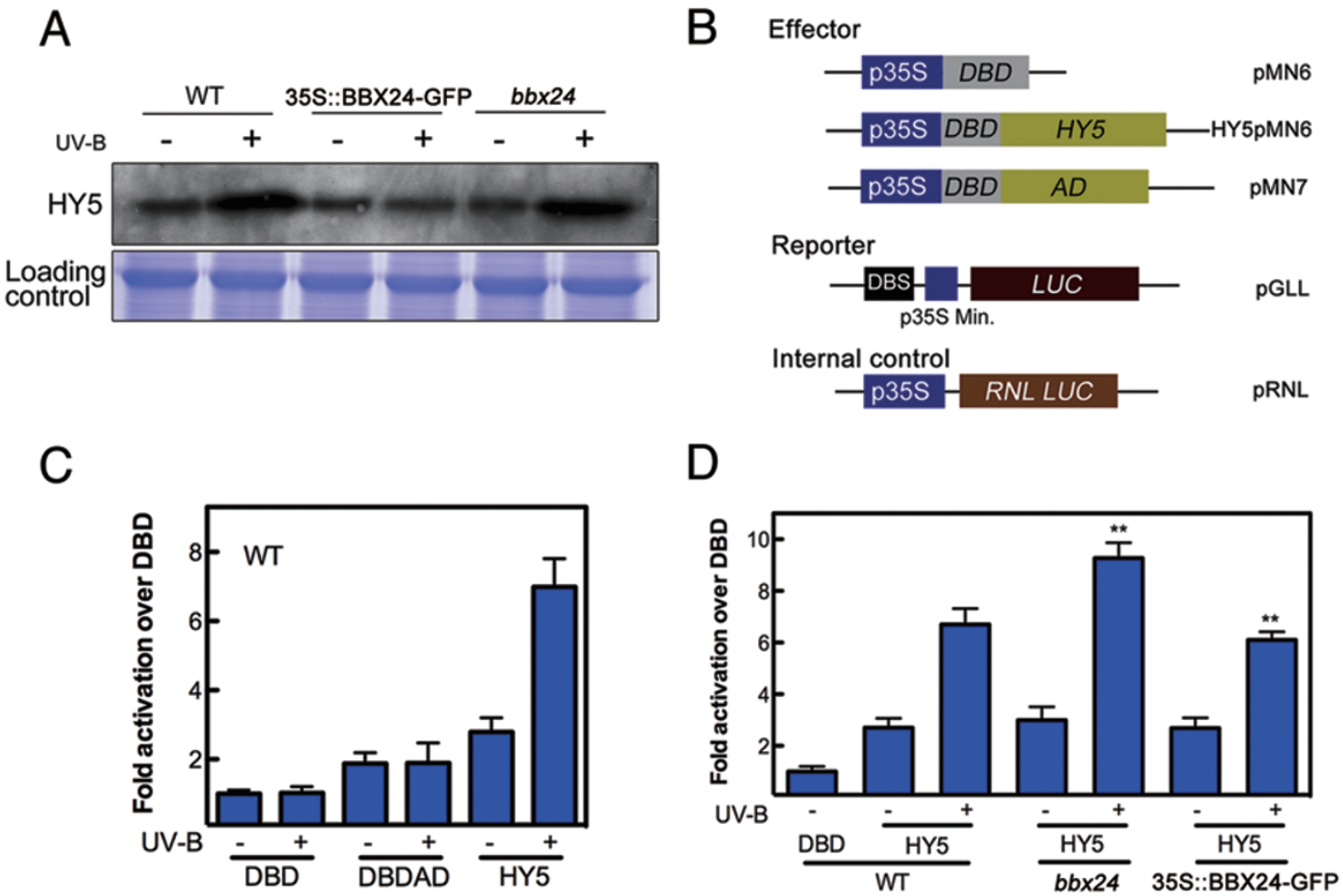

Figure 4 BBX24 suppresses UV-B-induced HY5 accumulation and negatively modulated HY5's transcriptional activation activity. (A) UV-B-induced HY5 accumulation in wild type, bbx24 and 35S::BBX24-GFP plants. Protein extracts were immunoprobed with a HY5 antibody. (B) Constructs used for the HY5 transcriptional activation activity. AD, GAL4 activation domain; DBD, GAL4 DNA-binding domain; DBS, GAL4 DNA-binding site; LUC, firefly luciferase; RNL LUC, Renilla luciferase. Protoplasts were extracted from the leaves of four-week-old wild type, bbx24 and 35S::BBX24-GFP plants, and transformed with effector constructs constitutively expressing a DBD-HY5 fusion (DBDHY5), DBDAD or DBD alone. The protoplasts were treated with supplementary UV-B radiation $\left(0.6 \mathrm{~W} \mathrm{~m}^{-2}\right)$ for $6 \mathrm{~h}$. (C, D) Transcriptional activity was measured with the dualLuciferase assay system (Promega). Data shown are means \pm SEM of four independent replications. Statistically significant differences of $b b \times 24$ or 35 S::BBX24-GFP relative to wild type are indicated by a double asterisk $(P<0.01)$. 
environmental signal for which plants have evolved specific and sensitive UV-B perception systems. Recently, significant progress has been made in our understanding of the UV-B response pathway in Arabidopsis, which involves the UV-B photoreceptor UVR8 [9], two positive regulators COP1 [12] and HY5 [23], and two WD40-repeat-containing proteins, RUP1 and RUP2 that negatively regulate the pathway [24]. In this study, we identified BBX24 as a new negative regulator of UV-B responses in Arabidopsis.

$B B X 24$ is a potent regulator of photomorphogenic $U V-B$ responses

The Arabidopsis BBX24 protein was originally named SALT TOLERANCE for its ability to confer salt tolerance when overexpressed in yeast [15]. Later it was found to function as a negative regulator of light-regulated photomorphogenesis [16]. In this study, we demonstrated that BBX24 also acts negatively in photomorphogenic UV-B responses. The $b b \times 24$ mutant is sensitive to the narrowband $(311 \mathrm{~nm})$ UV-B radiation and exhibited a dwarf phenotype after 5 days' UV-B treatment, compared to the wild-type control (Figure 1A). By contrast, BBX24 overexpression transgenic plants have much weaker responses to UV-B than the $b b \times 24$ and wild-type plants (Figure 1A and 1B). BBX24 is also involved in UV-B-regulated anthocyanin biosynthesis, which is significantly enhanced in the $b b \times 24$ mutant but reduced in BBX24 overexpression lines (Figure 1C). These results clearly point to a negative role of BBX24 in UV-B inhibition of hypocotyl elongation and UV-B enhancement of anthocyanin biosynthesis.

In addition to the hypocotyl and anthocyanin responses that are regulated by both light and UV-B signals, we also observed some UV-B-specific phenotypes in the bbx24 mutant, including changes in primary root elongation (Figure 1F, Supplementary information, Figures S3 and S9) and chlorophyll biosynthesis (Supplementary information, Figure S10). The increased inhibition of primary root elongation in $b b \times 24$ seedlings is confined to $\mathrm{UV}-\mathrm{B}$, suggesting that BBX24 also plays a crucial role in UV-B-mediated inhibition of root elongation. Therefore, BBX24 and the recently identified RUS1 and RUS2 proteins for root UV-B response $[25,26]$ represent part of the UV-B sensing machinery in Arabidopsis roots. The root elongation is significantly blocked in rus $1-1$ and rus 2-1 mutants exclusively under very-low-fluence UV-B $[25,26]$. Interestingly, this phenotype of rus $1-2$ under UV-B is not correlated with the common UV-Bresponsive genes, such as CHS and HY5 [26], indicating that the UV-B signaling pathway in the root is different from that in the shoot.
$B B X 24$ is a key $U V-B$ signaling component that interacts with both COPI and HY5

COP1 is a key signaling component in both light- and UV-B-regulated photomorphogenesis [12, 27]. Previous studies have shown that COP1 interacts with BBX24 in yeast [20], and the two proteins are co-localized in the nucleus [16]. However, there has been no convincing evidence supporting their direct interaction in planta. Our co-IP results clearly showed that COP1 interacts with BBX24 in vivo in a UV-B-dependent manner, and this requires UV-B-induced COP1 accumulation (Figure 2A). COP1 was assumed to mediate BBX24 degradation in the dark [16], but move away from nuclei in the presence of light [28]. Here, we provided solid evidence showing that the degradation of BBX24 in the dark is mediated by COP1 through the $26 \mathrm{~S}$ proteasome pathway. However, COP1 remains in the nucleus under UV-B radiation $[8$, 12], and our results show that BBX24 accumulates at the same time, suggesting that COP1 does not function as an E3 ubiquitin ligase that targets BBX24 in UV-B responses. This notion was further supported by the evidence that MG132 treatment has little effect on the UVB-induced BBX24 accumulation (Figure 2B). These results are in agreement with a previous finding that both COP1 and HY5 accumulated in the nucleus in response to UV-B [12]. Furthermore, UV-B-induced BBX24 protein level is dramatically reduced in the cop 1-4 background (Figure 2B), suggesting that COP1 is required for BBX24 accumulation. Taken together, all these results indicate that the function of COP1 in UV-B responses is very different from that in light signaling.

The genetic relationship of BBX24 and COP 1 in UV-B responses is also different from that in light signaling. The copl-4 is a weak mutant in response to light, but can be regarded as a null mutant in UV-B signaling [12]. Overexpression of $B B X 24$ partially suppressed the cop 1-4 phenotype under the red light and $b b \times 24$ showed enhanced light sensitivity in the cop 1-4 background [16], suggesting that the function of BBX24 in light responses is partially independent of COP1. However, our genetic results show that the longer hypocotyl and the lower anthocyanin content of cop 1-4 under UV-B is suppressed by bbx24 but not completely suppressed by $35 \mathrm{~S}: \mathrm{BBX} 24-\mathrm{GFP}$, indicating that the activity of COP1 in UV-B signaling partially depends on BBX24.

We have also demonstrated that BBX24 interacts with HY 5 both in vitro and in vivo, indicating that the two proteins work together too. Our genetic results showed that overexpression of $B B X 24$ in hy5-215 resulted in a clearly longer hypocotyl than either hy5-215 or $35 \mathrm{~S}$ BBX24 (Figure 3C). This suggests that BBX24 has activities that are independent of HY5. Interestingly, the 
phenotypes of the hy5-215 bbx24 homozygous mutant are reminiscent of those of wild type under UV-B (Figure $3 \mathrm{C}$ and $3 \mathrm{D}$ ), suggesting that HY5 and BBX24 act antagonistically in UV-B response. We were also able to demonstrate that BBX24 reduces HY5 protein accumulation and represses its transcriptional activation activity in vivo (Figure 4). These results provide a molecular basis for BBX24 function in UV-B signaling.

BBX24 may function as a new component of the negative feedback regulatory module in UV-B signaling

In light signaling, the action of positive signaling factors is counterbalanced by a set of important repressor proteins, including COP1 and the four members of the SUPPRESSOR OF PHYA-105 (SPA) gene family, which interact and form complexes in vivo [29]. BBX24 was believed to form a negative regulatory network with these repressor proteins to fine-tune light-regulated gene expression and thereby preventing an exaggerated light response [16]. However, how BBX24 interacts with these repressors to regulate the light responses is currently unknown.

Plant responses to UV-B are largely mediated by the UVR8-COP1-HY5 pathway [5]. UVR8, COP1 and HY5 are positive regulators of UV-B signaling and their functions must be balanced by a set of repressor proteins to prevent overstimulation by UV-B. However, to date few negative regulators of UV-B responses have been identified except for RUP1 and RUP2, two WD40-repeatcontaining proteins in Arabidopsis that were believed to function in a negative feedback loop downstream of UVR8-COP1 by interacting with UVR8 [24]. However, it remains unclear how the UVR8-RUP interaction results in repression of UV-B responses.

The expression pattern of BBX24 induced by UV-B is similar to that of RUPs, but we did not detect a direct interaction between UVR8 and BBX24 in the yeast twohybrid system, either with or without the UV-B treatment (Supplementary information, Figure S11). Instead, BBX24 strongly interacts with both COP1 and HY5 and $\mathrm{COP} 1$ is required for $\mathrm{BBX} 24$ accumulation in response to UV-B (Figures 2 and 3). These results suggest a different mode of function of BBX24 in UV-B signaling. Based on our results in this study, we propose a model for BBX24 function in UV-B signaling (Figure 5). Briefly, the UV-B signal is perceived by the UV-B receptor UVR8, resulting in rapid accumulation of COP1 and its interaction with UVR8. UVR8-COP1 interaction presumably stabilizes and activates HY5 for its transcriptional activity, leading to UV-B-regulated gene expression and photomorphogenesis [8]. This UV-B signaling cascade is feedback regulated by a set of negative regulators, which

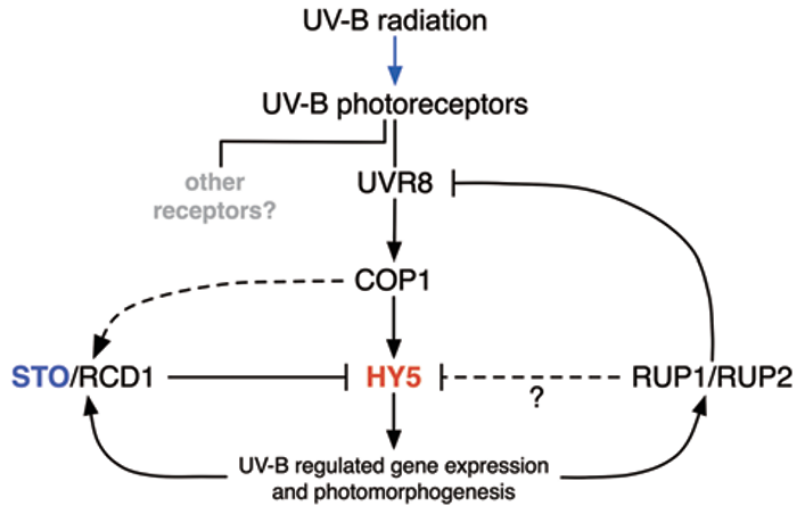

Figure $5 \mathrm{~A}$ model for BBX24 function in plant UV-B responses. The UV-B radiation is specifically perceived by the UV-B photoreceptor UVR8, resulting in the rapid accumulation of COP1 and its interaction with UVR8. COP1-UVR8 interaction presumably stabilizes and activates HY5 for its transcriptional activity, leading to UV-B-regulated gene expression and photomorphogenesis. This UV-B signaling cascade is feedback regulated by a few negative regulators, including BBX24, RCD1, and RUP1/ RUP2. While RUP1/RUP2 negatively regulate UV-B signaling by interacting with UVR8 [24], the BBX24 protein fine-tunes the UV-B responses by impinging on HY5. The BBX24-interacting protein RCD1 [19] may also play a role in the BBX24 feedback regulatory module.

may include STO/BBX24, RCD1, and RUP1/RUP2. While RUP1/RUP2 negatively regulate UV-B signaling by interacting with UVR8 [24], the BBX24-RCD1 complex may fine-tune the UV-B responses by interacting with both COP1 and HY5. We hypothesize that COP1BBX24 interaction stabilizes the BBX24 protein and BBX24-HY5 interaction results in reduced accumulation and/or transcriptional activity of HY5. Since HY5 is transcriptionally regulated by UVR8 [24], it is tempting to speculate that the RUPs-UVR8 interaction may also attenuate the HY5 activity and/or accumulation. If this is the case, it will suggest a central role of HY5 in regulating UV-B responses.

\section{Materials and Methods}

\section{Plant growth, light and UV-B treatments}

Wild type and mutant Arabidopsis plants used in this study were all in Col-0 ecotype background. Triple mutants of bbx24 with different photoreceptor mutants were made by crossing bbx 24 to the double mutants of phyA-211 phyB-9, phot1-5 phot21 , and cry1-104 cry2-1 [36, 37]. The F2 seedlings were screened under either blue or red light, and seedlings with long hypocotyls were selected according to previous phenotypic analysis [16]. The homozygous phot 1 phot 2 bbx 24 triple mutants were further confirmed by PCR and phenotypic analysis in F3 and F4, while the 
homozygous phyA phyB bbx24 and cryl cry2 bbx24 triple mutants were verified in F6 and F7.

Seeds were surface sterilized and sown on $1 / 2$ Murashige and Skoog medium containing 1\% sucrose for germination and growth under appropriate light conditions for 5 to 7 days at $22{ }^{\circ} \mathrm{C}$. Blue, red, or far-red light was supplied by LED light sources (Percival, IA, USA), with fluence rates of $9 \mu \mathrm{mol} \mathrm{m} \mathrm{m}^{-2}, 45 \mu \mathrm{mol} \mathrm{m} \mathrm{m}^{-2} \mathrm{~s}^{-1}$, and $20 \mu \mathrm{mol} \mathrm{m} \mathrm{m}^{-2} \mathrm{~s}^{-1}$, respectively (measured with Quantitherm Light Meter, Hansatech, England). White light was supplied by cool-white fluorescent lamps (Philips TLD30W/865 tubes, 10 $\mu \mathrm{mol} \mathrm{m} \mathrm{m}^{-2} \mathrm{~s}^{-1}$; Quantitherm Light Meter). The UV-B treatment was performed by narrowband UV-B tubes (Philips PLS9W/01, 0.6 W $\mathrm{m}^{-2}$; measured with iHR550 spectroradiometer, Horiba JobinYvon, Japan; approximately $1.57 \mu \mathrm{mol} \mathrm{m} \mathrm{m}^{-2} \mathrm{~s}^{-1}$ ), supplemented with white light. UV-B radiation from the lamps was filtered through cellulose acetate film to block potential UV-C (+UV-B). Radiation for control plants was filtered through Mylar film to remove both UV-B and UV-C (-UV-B).

\section{Vector construction}

To generate $35 \mathrm{~S}:: \mathrm{BBX} 24-\mathrm{GFP}$ transgenic plants, a full-length $B B X 24$ cDNA fragment was cloned by RT-PCR using the primer pair BBX35SF (5'-GTCGACGATGAAGATACAGTGTGATGT-3') and BBX35SR (5'-GGATCCCGGCCAAGATCAGGGACAATG-3'). To generate pBBX24::BBX24-GFP transgenic plants, the genomic sequence of $B B X 24$ was cloned by PCR from Arabidopsis genomic DNA using the primer pair BBXF (5'-AAGAATTCGTGTTTTGGGAGTTATTAACTTC-3') and BBXR (5'-CCATGGTGCCAAGATCAGGGACAATGAAGTG-3'). The PCR products were cloned into pMD19-T Simple vector (TaKaRa, Dalian, China) to generate pMD19-BBX24 and pMD19-pBBX24BBX24. The pMD19-BBX24 was digested with SalI and BamHI, and subcloned to pBEGFP vector to produce $35 \mathrm{~S}: \mathrm{BBX} 24-\mathrm{GFP}$. The pMD19-pBBX24-BBX24 was digested with EcoRI and NcoI, and subcloned to pCAMBIA1302 to produce pBBX24::BBX24GFP. The pCAMBIA1302 vector was pre-treated to remove its $35 \mathrm{~S}$ promoter.

\section{Extraction and quantification of anthocyanins}

The anthocyanins were extracted and quantified as previously described [30]. Briefly, seedlings were harvested, quickly weighed, and placed into tubes containing extraction solution (18\% 1-propanol and $1 \% \mathrm{HCl}$ ). The tubes were placed in boiling water for $3 \mathrm{~min}$ and then left in darkness for at least $3 \mathrm{~h}$ at room temperature. After a brief centrifugation to pellet the tissue, the solution was removed and diluted with the extraction solution. The anthocyanins were quantified spectrophotometrically and the results were reported as $\mathrm{A}_{535}-2\left(\mathrm{~A}_{650}\right) \mathrm{g}^{-1}$ fresh weight.

\section{RNA extraction and real-time $q P C R$ analysis}

Seedlings were harvested and ground immediately in liquid nitrogen. Total RNA was extracted with E. Z. N. A. Plant RNA Kit (Omega, USA) according to the manufacturer's instruction. Following RNA extraction, a DNase I treatment (RNA-free; Promega, Madison, WI, USA) was used to eliminate genomic DNA contamination. Complementary DNA was then synthesized using PrimeScript 1st strand cDNA synthesis kit (Takara Biotechnology, Dalian, China). Real-time PCR was conducted according to the Power SYBR Green PCR manual (Applied Biosystem, Foster
City, CA, USA). PCR was performed on the ABI PRISM 7300 Sequence Detection System (Applied Biosystems) using Power SYBR Green Master Mix following the recommended conditions $\left(2 \min 50{ }^{\circ} \mathrm{C}, 10 \min 95^{\circ} \mathrm{C}, 40 \times\left(15 \mathrm{~s} 95{ }^{\circ} \mathrm{C}\right.\right.$; $\left.\left.1 \min 60{ }^{\circ} \mathrm{C}\right)\right)$. All primers were listed in Supplementary information, Table S1. Primer pair efficiencies were estimated by analysis of the amplification curves with the LinReg software [31], and the average efficiency of all reactions on a plate (which was always $>1.95$ ) was used in calculations. Each quantification assay includes at least three biological replicates. To normalize the qPCR data, three or four reference genes [32] were used in each experiment. Reference genes for detecting $B B X 24$ gene expression in wild type were $E 2 F 1 a$ and $U B Q 5$. Reference genes for detecting gene expression in wild type, $b b \times 24$ and 35S::BBX24 were E2F1a and eIF4A.

\section{Protein-protein interaction assays}

The yeast two-hybrid assay was performed as described before [33]. Briefly, full-length of HY5 cDNA, HY5 $\triangle$ bZIP (lacking bZIP domain) or bZIP (only containing HY5 bZIP domain) were cloned into the pGBKT7 bait vector (Clontech, CA, USA) in-frame with the GAL4-DNA-binding domain. The $B B X 24$ cDNA fragments were cloned into the pGADT7 prey vector in-frame with the GAL4 activation domain. Test constructs were transformed into yeast strain AH109, and interactions were selected by minimal medium/-Trp/-Leu/-Ura/-His plates following the instructions of the manufacturer (Clontech).

The Co-IP experiment was carried out to confirm the interaction of BBX24 with either COP1 or HY 5 in vivo by following the procedure of Serino and Deng [34]. Briefly, total protein was extracted by the IP buffer $(150 \mathrm{mM} \mathrm{NaCl}, 50 \mathrm{mM}$ Tris-HCl, $\mathrm{pH} 7.5$, $0.1 \%$ NP-40, $5 \mathrm{mM}$ DTT, and 1 piece of complete protease inhibitor tablet). The protein extracts were precipitated with a monoclonal anti-GFP antibody (Santa Cruz Biotechnology) immobilized to the Protein A and G-PLUS Agarose beads (Santa Cruz Biotechnology) overnight at $4{ }^{\circ} \mathrm{C}$, and the beads were then washed three times in IP buffer. Total proteins and immunoprecipitates were analyzed by western blotting by using either an anti-COP1 or antiHY5 antibody (Santa Cruz Biotechnology) to detect the COP1BBX24 or HY5-BBX24 interaction.

\section{In vivo transcriptional transactivation assay}

HY5 transcriptional activation activity was analyzed as previously described [35]. Briefly, the HY5 cDNA fragment was cloned into the pMN6 vector, in frame with the GAL4-DNA-binding domain. The pMN6 vector alone was used as a negative control. Renilla Luciferase driven by $35 \mathrm{~S}$ promoter was used as an internal control. These vectors were co-transformed into wild type, $b b \times 24$ or $35 \mathrm{~S}:$ :BBX24-GFP protoplasts. The protoplasts were treated with UV-B radiation $\left(0.6 \mathrm{~W} \mathrm{~m}^{-2}\right)$ for $6 \mathrm{~h}$. The signals of firefly and Renilla luciferases were detected by the Dual-Luciferase System (Promega).

\section{Acknowledgments}

We thank Dr Marta Rodriguez-Franco (University of Freiburg, Germany) for kindly providing the sto/bbx24 mutant, Dr Magnus Holm (University of Gothenburg, Sweden) for hy5-215, cop 1-4 mutants, Prof Winslow Briggs (Carnegie Institution for Science, USA) for various photoreceptor double mutants and valuable com- 
ments, Prof Peter Quail (University of California, Berkeley, USA) for vectors for transactivation assay, and Prof Chenwei Yang (South China Normal University, China) for the pBEGFP vector. This work was supported by grants from the National Natural Science Foundation of China (31070242) and the Research Fund for the Doctoral Program of Higher Education of China (20070574003, 20114407110006) to SSL, and grants from the Research Grant Council of Hong Kong $(465009,465410)$ to JXH.

\section{References}

1 Brosché M, Strid Å. Molecular events following perception of ultraviolet-B radiation by plants. Physiol Plant 2003; 117:110 .

2 Kim BC, Tennessen DJ, Last RL. UV-B-induced photomorphogenesis in Arabidopsis thaliana. Plant J 1998; 15:667674.

3 Jenkins GI, Brown BA. UV-B perception and signal transduction. In: Whitlam GC, Halliday KJ, eds. Light and Plant Development. Oxford: Blackwell 2007:155-182.

4 Ulm R, Nagy F. Signalling and gene regulation in response to ultraviolet light. Curr Opin Plant Biol 2005; 8:477-482.

5 Rizzini L, Favory JJ, Cloix C, et al. Perception of UV-B by the Arabidopsis UVR8 Protein. Science 2011; 332:103-106.

6 Kliebenstein DJ, Lim JE, Landry LG, Last RL. Arabidopsis $U V R 8$ regulates ultraviolet-B signal transduction and tolerance and contains sequence similarity to human Regulator of Chromatin Condensation 1. Plant Physiol 2002; 130:234-243.

7 Jenkins GI. Signal transduction in responses to UV-B radiation. Annu Rev Plant Biol 2009; 60:407-431.

8 Favory JJ, Stec A, Gruber H, et al. Interaction of COP1 and UVR8 regulates UV-B-induced photomorphogenesis and stress acclimation in Arabidopsis. EMBO J 2009; 28:591-601.

9 Brown BA, Cloix C, Jiang GH, et al. A UV-B-specific signaling component orchestrates plant UV protection. Proc Natl Acad Sci USA 2005; 102:18225-18230.

10 Yi CL, Deng XW. COP1 - from plant photomorphogenesis to mammalian tumorigenesis. Trends Cell Biol 2005; 15:618625 .

11 Osterlund MT, Hardtke CS, Wei N, Deng XW. Targeted destabilization of HY5 during light-regulated development of Arabidopsis. Nature 2000; 405:462-466.

12 Oravecz A, Baumann A, Mate Z, et al. CONSTITUTIVELY PHOTOMORPHOGENIC1 is required for the UV-B response in Arabidopsis. Plant Cell 2006; 18:1975-1990.

13 Griffiths S, Dunford RP, Coupland G, Laurie DA. The evolution of CONSTANS-like gene families in barley, rice, and Arabidopsis. Plant Physiol 2003; 131:1855-1867.

14 Khanna R, Kronmiller B, Maszle DR, et al. The Arabidopsis B-Box Zinc Finger Family. Plant Cell 2009; 21:3416-3420.

15 Lippuner V, Cyert MS, Gasser CS. Two classes of plant cDNA clones differentially complement yeast calcineurin mutants and increase salt tolerance of wild-type yeast. $\mathrm{J}$ Biol Chem 1996; 271:12859-12866.

16 Indorf M, Cordero J, Neuhaus G, Rodriuez-Franco M. Salt tolerance (STO), a stress-related protein, has a major role in light signalling. Plant J 2007; 51:563-574.

17 Yan H, Marquardt K, Indorf M, et al. Nuclear localization and interaction with COP1 are required for STO/BBX24 function during photomorphogenesis. Plant Physiol 2011; 156: 17721782

18 Jiang L, Wang Y, Björn LO, Li S. Arabidopsis RADICALINDUCED CELL DEATH1 is involved in UV-B signaling. Photochem Photobiol Sci 2009; 8:838-846.

19 Jaspers P, Blomster T, Brosche M, et al. Unequally redundant RCD1 and SRO1 mediate stress and developmental responses and interact with transcription factors. Plant J 2009; 60:268279.

20 Holm M, Hardtke CS, Gaudet R, Deng XW. Identification of a structural motif that confers specific interaction with the WD40 repeat domain of Arabidopsis COP1. EMBO J 2001; 20:118-127.

21 Datta S, Hettiarachchi C, Johansson H, Holm M. SALT TOLERANCE HOMOLOG2, a B-Box protein in Arabidopsis that activates transcription and positively regulates light-mediated development. Plant Cell 2007; 19:3242-3255.

22 Datta S, Johansson H, Hettiarachchi C, et al. LZF1/SALT TOLERANCE HOMOLOG3, an Arabidopsis B-box protein involved in light-dependent development and gene expression, undergoes COP1-mediated ubiquitination. Plant Cell 2008; 20:2324-2338.

23 Ulm R, Baumann A, Oravecz A, et al. Genome-wide analysis of gene expression reveals function of the bZIP transcription factor HY5 in the UV-B response of Arabidopsis. Proc Natl Acad Sci USA 2004; 101:1397-1402.

24 Gruber H, Heijde M, Heller W, Albert A, Seidlitz HK, Ulm R. Negative feedback regulation of UV-B-induced photomorphogenesis and stress acclimation in Arabidopsis. Proc Natl Acad Sci USA 2010; 107:20132-20137.

25 Leasure CD, Tong HY, Yuen GG, Hou XW, Sun XF, He ZH. ROOT UV-B SENSITIVE2 acts with ROOT UV-B SENSITIVE1 in a root ultraviolet B-sensing pathway. Plant Physiol 2009; 150:1902-1915.

26 Tong H, Leasure CD, Hou X, Yuen G, Briggs W, He Z-H. Role of root UV-B sensing in Arabidopsis early seedling development. Proc Natl Acad Sci USA 2008; 105:21039-21044.

27 Holm M, Ma LG, Qu LJ, Deng XW. Two interacting bZIP proteins are direct targets of COP1-mediated control of lightdependent gene expression in Arabidopsis. Genes Dev 2002; 16:1247-1259.

28 von Arnim AG, Deng XW. Light inactivation of Arabidopsis photomorphogenic repressor COP1 involves a cell-specific regulation of its nucleocytoplasmic partitioning. Cell 1994; 79:1035-1045.

29 Zhu D, Maier A, Lee JH, et al. Biochemical characterization of Arabidopsis complexes containing CONSTITUTIVELY PHOTOMORPHOGENIC1 and SUPPRESSOR OF PHYA proteins in light control of plant development. Plant Cell 2008; 20:2307-2323.

30 Noh B, Spalding EP. Anion channels and the stimulation of anthocyanin accumulation by blue light in Arabidopsis seedlings. Plant Physiol 1998; 116:503-509.

31 Ramakers C, Ruijter JM, Deprez RH, Moorman AF. Assumption-free analysis of quantitative real-time polymerase chain reaction (PCR) data. Neurosci Lett 2003; 339:62-66.

32 Czechowski T, Stitt M, Altmann T, Udvardi MK, Scheible WR. Genome-wide identification and testing of superior reference genes for transcript normalization in Arabidopsis. 
Plant Physiol 2005; 139:5-17.

33 He JX, Gendron JM, Yang Y, Li J, Wang ZY. The GSK3-like kinase BIN2 phosphorylates and destabilizes BZR1, a positive regulator of the brassinosteroid signaling pathway in Arabidopsis. Proc Natl Acad Sci USA 2002; 99:10185-10190.

34 Serino G, Deng X-W. Protein coimmunoprecipitation in Arabidopsis. Cold Spring Harb Protoc 2007; 2007:pdb.prot4683. doi:10.1101/pdb.prot4683

35 Huq E, Al-Sady B, Hudson M, Kim C, Apel K, Quail PH.
PHYTOCHROME-INTERACTING FACTOR 1 is a critical bHLH regulator of chlorophyll biosynthesis. Science 2004; 305:1937-1941.

36 Cerdan PD, Chory J. Regulation of flowering time by light quality. Nature 2003; 423:881-885.

37 Mao J, Zhang YC, Sang Y, Li QH, Yang HQ. A role for Arabidopsis cryptochromes and COP1 in the regulation of stomatal opening. Proc Natl Acad Sci USA 2005; 102:12270-12275.

(Supplementary information is linked to the online version of the paper on the Cell Research website.) 